\title{
Les défis de la traduction du théâtre multilingue: le cas de Requiem for the Last Kaiser
} Marcelline Massoua II, Université de Pretoria

\begin{abstract}
The translation of multilingual theatre presents challenges since it queries the generally accepted definition(s) and characteristics of translation as a process and as a product. To date, much research has been carried out with regards to multilingual translation and theatre translation separately, but less on both (multilingual theatre translation), thus doubling the challenge. The aim of this study is to examine the different strategies, techniques and thought processes involved in solving the challenges encountered in the translation of the multilingual play Requiem for the Last Kaiser (1998), written by the Cameroonian playwright Bate Besong and recounting the population's uprising against a dictatorship in a neo-colonial country. This study identifies these challenges and compares the strategies and techniques used to translate the multilingual occurrences in Requiem for the Last Kaiser to those currently recommended in Translation theory as well as by practitioners.
\end{abstract}

\section{Résumé}

La traduction du théâtre multilingue présente des défis puisqu'elle remet en question les définitions et les caractéristiques généralement acceptées de la traduction en tant que processus et produit. Plusieurs chercheurs se sont penchés sur la traduction multilingue et la traduction théâtrale séparément mais on rencontre peu l'association des deux (traduction théâtrale multilingue), ce qui ajoute au défi initial. Le but de cette étude est d'examiner les différentes stratégies, techniques et procédures mises en œuvre pour aborder les défis rencontrés dans la traduction de la pièce de thêâtre multilingue Requiem for the Last Kaiser (1998). Elle a été écrite par le dramaturge camerounais Bate Besong et raconte le 
soulèvement de la population d'un État néocolonial contre son pouvoir dictatorial. La recherche s'est faite en identifiant ces défis et en comparant les stratégies et techniques effectivement utilisées dans la traduction des occurrences multilingues de Requiem for the Last Kaiser et celles préconisées par les théories traductologiques et les praticiens.

\section{Keywords}

Bate Besong

traduction théâtrale

multilingue

traduction multilingue

stratégies de traduction

Lorsque l'on parle de la littérature camerounaise, on fait spontanément référence à une 'écriture camerounaise d'expression française'. La littérature de langue anglaise reste en marge car elle est 'cruellement peu représentée, mal représentée et parfois carrément exclue de nombre d'instances qui concourent directement ou indirectement à la reconnaissance autonome de toute littérature nationale' (Fandio 2001). La pièce de théâtre Requiem for the Last Kaiser du dramaturge camerounais d'expression anglaise, Bate Besong, dont la caractéristique principale est la présence de multiples langues dans le même texte, met en contexte la collaboration linguistique dans le domaine littéraire au Cameroun. Face à ce développement contrasté et dans le souci de susciter une véritable littérature nationale riche de ses deux pans linguistiques, la traduction dans son sens propre et métaphorique s'avère être un moyen permettant de développer des échanges littéraires et d'établir des contacts entre systèmes littéraires. La traduction peut jouer un rôle capital dans le développement de la 
communication interculturelle et pourrait par conséquent servir dans le contexte camerounais. L'œuvre de Besong a d'autant plus d'intérêt qu'elle présente deux aspects de la littérature que l'on retrouve rarement associés, notamment la littérature multilingue et le théâtre.

Le projet de traduction de Requiem for the Last Kaiser a présenté de nombreux défis à plusieurs niveaux; ces défis étant liés à la nature même du texte et aux moyens à mettre en œuvre pour sa traduction effectuée, entre autres, dans l'optique d'entamer le dialogue au sein de la littérature camerounaise. Cependant, la documentation disponible ne permettait pas de prendre des décisions traductionnelles argumentées purement théoriques. En effet, la recherche en traduction théâtrale a connu ces dernières années un dynamisme qui contraste avec le peu d'attention qu'on y avait accordé dans le passé par comparaison avec la traduction d'autres types de textes littéraires. Les années 1970 et 1980 constituent un vivier de contributions qui nourrissent les connaissances théoriques disponibles en traduction théâtrale complétées par les récentes parutions. ${ }^{1}$ De la même manière et séparément, la traduction multilingue a fait couler beaucoup d'encre et continue à susciter l'intérêt des chercheurs étant donné que les sociétés plurilingues ne sont plus l'exception mais elles sont devenues la norme comme l'atteste William Mackey (1993: 46), ce qui explique la présence de plus en plus fréquente de textes multilingues. Cependant, les chercheurs ne se sont pas beaucoup intéressés à la traduction théâtrale multilingue et à ses spécificités.

Cette étude se donne donc pour but de se pencher sur la traduction des pièces de théâtre multilingues et plus particulièrement sur la traduction de Requiem for the Last Kaiser. Les limites de cette étude ne nous permettront pas d'aborder la réception de la pièce par le public cible. Par contre, nous nous concentrerons sur les différents défis rencontrés dans la traduction de l'œuvre, les normes et les conventions mises à la disposition du traducteur de pièces de théâtre et du traducteur de textes multilingues et enfin nous examinerons en 
comparaison les stratégies techniques et procédures effectivement utilisées pour la traduction partielle de Requiem for the Last Kaiser.

\section{Analyse documentaire}

Il convient de se pencher sur les normes et conventions stratégiques qui régissent théoriquement la traduction théâtrale et la traduction multilingue séparément pour en tirer des stratégies, des techniques et des procédures applicables a priori à la traduction de la pièce de théâtre Requiem for the Last Kaiser. La problématique de la traduction et plus particulièrement de la traduction littéraire se rapporte non seulement à la fidélité aux idées de l'auteur mais à son style, à son utilisation du langage et à la réception qui en sera faite par un autre public. Le traducteur doit donc conserver le style de l'auteur en travaillant son langage. Il s'agit de créer une communication entre l'œuvre et le récepteur pour lui transmettre un message et produire chez lui un effet (Zhang 2006: 106). Le but n'est pas toujours d'instruire mais on ne sort jamais de la lecture d'une œuvre complètement inchangé.

Pour ce qui est du théâtre spécifiquement, il s'agit d'un genre littéraire qui concilie à la fois littérature et spectacle. Le théâtre n'est pas seulement perçu comme un lieu où l'on regarde comme le suggère l'origine grecque theatron, mais également comme un lieu où l'on vit. Pour Gaoussou Diawara, universitaire et dramaturge malien, le théâtre en Afrique soulage les hommes de la souffrance et n'est pas utopique dans son ambition de refaire le monde, de repenser les erreurs pour mener au développement (Diawara 2010). Dans cette perspective, le théâtre présente un rôle social, celui de plaider pour la communication directe entre les individus, pour leur liberté de penser, de sentir et de réagir immédiatement au discours qu'on leur propose afin de réhabiliter le hasard et l'insécurité et de ramener 'au-devant de la scène' les droits et les exigences de l'imagination (Ronfard 1985: 228). Cette conception place le public au centre du théâtre et rend essentielle son interaction avec la scène. Ce qui fait donc la 
théâtralité d'une œuvre dramatique est qu'elle est destinée à être représentée, elle n'est pas écrite pour un lecteur mais pour un acteur qui parle et qui joue devant un public. Le déchiffrage d'une œuvre théâtrale suscite de l'imagination ainsi que l'activation de processus mentaux chez le public, sans oublier un plaisir véritable.

\section{Spécificités et stratégies en traduction théâtrale}

Avec la traduction théâtrale, les défis de la traduction des textes littéraires prennent une nouvelle dimension de complexité, car le texte n'est ici qu'un élément de la totalité du discours théâtral (Bassnett 2008: 130). Il faut y ajouter le spectacle. Historiquement, la traduction du théâtre a donné une importance variée au texte théâtral et à sa représentation. Le traducteur doit décider s'il considèrera l'œuvre comme un texte théâtral assimilable à n'importe quel texte littéraire ou comme un texte spectaculaire orienté vers la représentation. Dans cette dernière catégorie, le traducteur collabore avec le metteur en scène et les acteurs car ils sont tous des intermédiaires entre le texte théâtral et le public. Il a donc fallu, pour les besoins de cette étude, nuancer ces approches liées au texte théâtral car le traducteur devrait traduire non seulement le contexte mais aussi la gestuelle codée dans le langage qui contribue au travail de l'acteur. Si le traducteur ignore les systèmes qui s'ajoutent au système purement littéraire, il peut échouer dans sa tâche. L'étude de la pièce de théâtre Requiem for the Last Kaiser a révélé qu'une solution intermédiaire qui donne sa place au texte à traduire devrait être préconisée. Il s'agit de considérer le texte théâtral comme un texte dramatique c'est-àdire un texte écrit qui établit le lien entre écriture et techniques théâtrales. Ce choix implique de prendre en compte la relation entre les répliques et les indications scéniques; l'immédiateté du discours théâtral dont les allusions et les références doivent être connues de tous et facilement perceptibles par le public cible; et la jouabilité du texte qui doit rendre le théâtre vivant en établissant le lien entre la gestuelle et le choix, la musique et la diction des 
mots. La réalisation de toutes ces caractéristiques est difficile à atteindre pour le traducteur qui n'est ni dramaturge, ni metteur en scène. C'est pourquoi, les nouvelles théories de la traduction théâtrale ont redonné de l'importance au théâtre essentiellement comme texte littéraire qui prend en compte la complexité de la tâche du traducteur théâtral. L'un des chefs de file de ces théories, Susan Bassnett, affirme que le traducteur théâtral doit utiliser les mêmes stratégies que le traducteur littéraire qui travaille avec les incohérences du texte et qui laisse la résolution de ces incohérences à quelqu'un d'autre (Bassnett 1998: 107). La collaboration entre le traducteur et le metteur en scènede la pièce de théâtre reste utile mais la traduction doit respecter les difficultés et les ambigüités du texte original et permettre à la représentation de s'épanouir en une infinité de possibilités (Bassnett cité par Regattin 2004: 164). Selon Bassnett, le traducteur doit donc éviter d'offrir un texte unidirectionnel, gommé de toutes les spécificités et possibilités d'interprétations offertes par l'original. En fin de compte, l'enjeu de la traduction théâtrale est de produire un texte jouable mais peut-on y arriver sans lever l'ambigüité ?

La traduction théâtrale comporte une dimension culturelle en ce que le théâtre offre un moyen concret de visualiser les éléments abstraits de la culture source. Le théâtre est donc en-soi un moyen de traduire, de clarifier ce qui peut porter à confusion. Peter Brook affirme à cet effet: 'Here lies the responsibility of the theatre: what a book cannot convey, what no philosopher can try to explain, can be brought into our understanding by the theatre. Translating the untranslatable is one of its roles' (Cité par Zhang 2006: 140). Phyllis Zatlin partage cette idée selon laquelle la performance est importante en traduction théâtrale car la traduction des mots et des significations ne produira jamais un texte jouable. De manière pratique, le traducteur reste confronter à un dilemme, s'il accentue les particularités de l'étrangeté dans la traduction, il court le risque de rompre le lien entre le public et la représentation ou de rendre le texte illisible. D'autre part, si le traducteur décide d'adapter la 
culture d'origine en gommant l'étrangeté, considérant que la culture est universelle, l'étrangeté devient invisible. Ce problème se pose avec plus d'acuité pour la traduction des œuvres d'anciennes colonies, alors que Georges Mounin affirme que traduire c'est vaincre les résistances culturelles qu'un texte oppose à sa traduction, Kathryn Batchelor (2009) déplore que la traduction des œuvres africaines soit trop transparente et trop explicative par rapport à l'original. Même s'il a été observé que les traductions, en général, ont pour caractéristiques communes d'être plus claires et moins ambigües que l'original, Batchelor y voit une caractéristique de la traduction des textes littétaires multilingues. À cet effet, Sylvie Chalaye affirme qu'à la fin des années 1980, il y a eu une rupture avec le théâtre africain classique marquée par l'absence de revendication identitaire et géographique. ${ }^{2}$ Les nouveaux dramaturges se veulent d'abord artistes, ils sont Africains par hasard, leur africanité se traduit par le lien profond avec l'oralité qui imprègne leur œuvre, la rythmique, le phrasé et la langue. Ce changement de perspective se retrouve bien dans l'œuvre étudiée et accentue le besoin de trouver une langue qui ne trahisse pas l'intention de l'auteur.

Ainsi, la solution intermédiaire, celle qui a été préconisée pour la traduction de Requiem for the Last Kaiser, est d'arriver à traduire en ménageant proximité et éloignement, familiarité et étrangeté (Pavis cité par Zhang 2006: 140) selon la partie de texte spécifique à traduire. Lorsque nous considérons traduction et théâtre, nous savons qu'il ne suffit pas de rendre le texte disponible mais également et plus encore, de traduire de telle sorte que le texte étranger puisse être joué convenablement par des acteurs sur la scène et accepté par le public. C'est un parti difficile à prendre a priori car le risque demeure d'avoir trop expliqué, trop dilué l'étrangeté ceci d'autant plus qu'il s'agit d'un théâtre africain présenté grâce à des langues européennes (entre autres). Pour ce qui est de savoir si le public rejette ou accueille l'étrangeté des cadres anciens ou contemporains, Paul Schmidt (cité par Zatlin 2005: 23) affirme qu'une traduction doit comporter un caractère étranger seulement s'il existe déjà dans 
l'original. À la question comment traduire une pièce de théâtre, nous avons retenu la nécessité d'être fidèle à l'auteur sans être complètement servile au texte et de produire des résultats adaptés à la scène, c'est-à-dire à même de toucher le public. Les théories théâtrales mettent donc en exergue le caractère spécifique du texte théâtral à traduire qui, dans le cadre de notre étude, est également le multilinguisme.

\section{Les stratégies de traduction dans les textes multilingues}

L'intérêt pour l'écriture multilingue en littérature n'est pas nouveau. La théorie du dialogisme de Mikhaïl Bakhtine et ses Euvres en particulier avaient déjà établi le rôle et l'importance de la pluralité linguistique dans le développement du roman moderne européen en mettant l'accent sur le multilinguisme inhérent au texte littéraire. Mais Bakhtine s'intéressait surtout à l'hétérogénéité présente dans une seule langue et non pas nécessairement à la juxtaposition de plusieurs langues dans un même discours. C'est cette dernière forme d'hétérogénéité qui inspire les réflexions actuelles essayant de souligner le rôle de l'hétéroglossie littéraire dans le but de réduire l'hégémonie de l'utilisation d'une seule langue en littérature (Bandia 2008: 139). ${ }^{3}$ La majorité des locuteurs multilingues dans le monde sont devenus minoritaires dans la plupart des États, par conséquent, pour revendiquer leurs droits et affirmer leur autonomie, ils se livrent à des pratiques linguistiques et esthétiques de toutes sortes qui transparaissent en littérature. Il n'est donc pas étrange de retrouver une multitude de langues dans Requiem for the Last Kaiser en raison de la diversité linguistique qui caractérise le pays d'origine de son auteur.

Pour ce qui est de la traduction des œuvres multilingues, les théoriciens n'ont dévolu que quelques lignes au multilinguisme sans s'attarder sur les problèmes qui se présentent dans la traduction du texte multilingue. Steiner et Sternberg y ont fait référence succinctement et sans donner de solution. Pour la plupart pessimistes, ils ne pensent pas que 
les problèmes soulevés par le texte multilingue puissent être résolus (Stratford 2008). Derrida, Berman et même Schogt se sont résignés à conclure que les systèmes linguistiques peuvent tout rendre à l'exception de la pluralité des langues. L'hétérogénéité des langues tend à disparaître dans la plupart des traductions et finalement il n'y a pas de solution miracle et il faut se résigner et accepter qu'aucune théorie ne puisse efficacement aider le traducteur dont les choix sont purement pratiques (Stratford 2008: 463). Nous concluons à la suite de Stratford qu'établir des règles universelles pour la traduction des textes multilingues limite les traducteurs et qu'il leur revient de résoudre les problèmes individuellement.

Les choix qui se présentent aux traducteurs sont soient d'effacer l'hétérogénéité ce qui peut aboutir à des effets regrettables; comme déformer le texte au niveau culturel, stylistique et idéologique. Ces effets ont pu être remarqués dans le contexte belge de l'entre-deuxguerres. Du fait de la standardisation de la langue, le texte peut perdre ses qualités et sa cohérence. Malgré ses conséquences indésirables, l'homogénéisation reste souvent très populaire pour des raisons financières, parce que les textes homogènes sont plus faciles à comprendre et plus agréables à lire (Stratford 2008: 464). Par conséquent, ce n'est pas tant la nature du texte multilingue qui justifie une traduction multilingue mais les contextes de production et de réception (Stratford 2008: 468). En effet, bien que le texte multilingue présente des difficultés traductionnelles, la réalité de l'industrie de la traduction montre que ce n'est pas une tâche impossible. Ricœur (2004: 15) affirme à dessein que ce qui est inaccessible, ce n'est pas la traduction mais la perfection de la traduction.

Stratford propose une traduction en langues multiples qui représente mieux l'hybridité croissante de notre monde actuel. Deux traductrices ont suivi cette voie et ont adopté des procédures de traduction tout autant impopulaires. Luise von Flotow opte pour le calque dans sa traduction vers l'anglais du roman Das Leben ist eine Karawanserai hat zwei Türen au seiner kam ich rein aus der andren ging ich raus écrit par Emine Sevgi dans un mauvais 
allemand, une sorte de dialecte turco-allemand (von Flotow 2000: 68). Le calque est une traduction aussi littérale que possible et ici il se construit délibérément sur les irrégularités de l'anglais pour égaler ou reproduire d'une certaine manière les éléments du texte source (von Flotow 2000: 68). Nathalie Ramiere a également décidé de garder autant que possible les empreintes de la langue d'origine. Elle le fait grâce à l'emprunt dans sa traduction en français de The Widows of Suzette Mayr afin de conserver le côté étrange du texte original et préserver sa dimension humoristique (Stratford 2008: 467). Ainsi, gommer le multilinguisme dans la traduction de la pièce de théâtre Requiem for the Last Kaiser, devient-il peu attrayant. Les traductions de von Flotow et de Ramiere constituent une tentative de traduction multilingue et ont orienté la quête de solutions traductionnelles de cette étude bien que les textes originaux soient des romans et non pas des pièces de théâtre et que les langues en présence soient moins hétéroclites que dans Requiem for the Last Kaiser.

La traduction théâtrale et la traduction multilingue prises séparément s'accordent sur la nécessité de conserver l'ambiguïté et l'étrangeté issue du texte source. Elles conviennent respectivement qu'il faut laisser à la représentation théâtrale le soin de clarifier les aspects étranges; d'autre part, la traduction doit embrasser le caractère hybride et multilingue du monde actuel en utilisant des procédés permettant de calquer la spécificité linguistique du texte source. Il convient maintenant, après ces considérations théoriques, de se pencher sur l'œuvre qui fait l'objet de cette étude et d'en déterminer les caractéristiques avant d'aborder les stratégies effectivement utilisées pour sa traduction.

\section{L'œuvre, l'auteur et le contexte d'écriture}

Requiem for the Last Kaiser est une pièce de théâtre qui a été publiée au Cameroun en 1998 par une maison d'édition locale, Pressbook. Elle avait d'abord été publiée au Nigéria en 1991 et a valu à son auteur le prix de l'Association des auteurs nigérians (ANA). Elle relate le 
soulèvement de la population d'un État néocolonial, l'Agidigidi, et met aux prises deux idéologies distinctes et opposées. D’un côté, le président du pays soutenu par la clique dirigeante locale et leurs maîtres étrangers qui oppressent, exploitent et appauvrissent les masses de manière cruelle pour garantir leurs propres intérêts. De l'autre côté, les exploités et les opprimés qui sont représentés principalement par les personnages de la Femme et des deux diplômés au chômage, expriment leur mécontentement, mais survivent en maintenant la situation telle quelle, sans essayer d'effectuer des changements sociaux radicaux. Lorsque les oppressés et les exploités jusque-là en rangs dispersés se regroupent, ils se battent pour changer par des moyens drastiques cette stagnation latente du statut quo. Ils envahissent les rues pour se saisir du pouvoir détenu par la minorité tyrannique et le donner au peuple. Les mots seuls ne pouvant laver la souillure qui s'est répandue dans le pays à cause des méfaits du dictateur, seul son sang peut le purifier. La pièce s'achève comme elle a commencé, avec un cercueil sur la scène, lorsque le dictateur se suicide et que les masses s'emparent du pouvoir (Ambanasom 2000).

Requiem for the Last Kaiser se présente immédiatement comme un défi, d'abord pour la compréhension du message que son auteur veut transmettre, et ensuite à travers les structures peu communes qu'il utilise pour réaliser son intention d'écriture. Cette œuvre n'appartient pas de manière stricte et rigide à un genre littéraire précis. Alfred Matumamboh, qui a écrit la préface de la deuxième édition de Requiem for the Last Kaiser, décrit le théâtre de Besong comme un théâtre moderne. Il serait pourtant réducteur de le limiter à une telle classification car bien que certains éléments rappellent d'emblée le théâtre moderne et ses figures de proue, le théâtre de Besong s'en distance par l'addition d'autres caractéristiques. En lisant la pièce de théâtre, on note immédiatement des ressemblances avec le théâtre de l'absurde notamment avec Ubu Roi d'Alfred Jarry en ce qu'il mélange le comique et le tragique et rejette les formes traditionnelles. La scène austère possède aussi un caractère 
symbolique pour créer un choc chez le public. Les similitudes avec Ubu Roi s'étendent au cadre de l'histoire qui n'est pas assez précis à savoir, la Pologne et un État néocolonial qui peuvent se situer n'importe où, au décor passe-partout, et aux caractéristiques du personnage principal qui est stupide, vulgaire, ambitieux, cruel et facilement influençable. Les deux auteurs malmènent les Nobles et même la langue (française pour Ubu-Roi; française et anglaise pour Requiem for the Last Kaiser). La langue d'écriture est marquée par les multiples répétitions du personnage principal et inclut des insultes, des jurons, des expressions idiomatiques et des grossièretés. On rencontre beaucoup d'ironie et d'humour ainsi que des allusions à la religion pour critiquer les abus de pouvoir et l'arbitraire. De plus, cette satire politique est encore et toujours d'actualité.

Le théâtre de Besong n'épouse pas entièrement l'absurdité de la condition humaine et l'inadéquation de l'approche rationnelle, il va plus loin et ajoute une touche d'espoir à la réalité chaotique. Les différences avec le théâtre de l'absurde s'observent au niveau du nombre de personnages qui sont plus nombreux que ceux d'Ubu-Roi car Besong met surtout l'accent sur les Masses. Le dénouement quant à lui diverge également car dans Ubu-Roi le dictateur réussit à échapper à la vindicte populaire et le roi légitime récupère le pouvoir qui lui revient de droit tandis que dans Requiem for the Last Kaiser, le dictateur, n'ayant aucune autre issue, se suicide et le peuple s'empare du pouvoir.

Entre plaidoyer et expression dramatique, Requiem for the Last Kaiser pousse à aller chercher ailleurs ses spécificités, notamment dans l'histoire littéraire de son écriture. Néanmoins, il faudrait relever ce qui rend cette œuvre tout à fait hors du commun. Il y a une quinzaine de personnages à travers lesquels la diversité de la pièce de théâtre se révèle. Entre autres, le premier personnage par ordre d'entrée sur scène est SAR Baal Njunghu Akhikikriki, président et divinité de l'Agidigidi. Le nom qui lui a été attribué désigne la fausseté de sa fonction, il est très entouré et servi par de nombreux assistants dont il se méfie. 
L'un de ses fervents supporters est Holy Prophet AAA (Abednego Absolom Amougou) Atangana que l'auteur décrit comme Monsignor Marabout. Cette description contraste avec le nom du personnage qui est censé être saint alors que la fonction de marabout est chargée très négativement dans certaines sociétés africaines car il s'agit souvent de vendeurs d'illusions. Les personnages suivants font partie de la masse: La Femme qui réveille les consciences engourdies et suscite le soulèvement des populations en organisant des manifestations; elle pousse graduellement au changement politique. L'Étudiant, d'abord soumis et convaincu de la véracité de la propagande gouvernementale, écoute La Femme, prend conscience des contradictions du régime et se rebelle contre le pouvoir en place. Le bras armé de l'État est représenté par État-Major Andze Abossolo, qui torture et réduit au silence les citoyens trop zélés, sans poser de questions. Il y a également deux étrangers, notamment le Banquier suisse et l'Ambassadeur Cracker Crookster. Ces deux étrangers sont associés au président, leurs rapports avec lui sont pourtant basés sur de faux semblants et de la fausse humilité. Poet as Mandela est un fou supposé qui sort des maximes et des paroles pleines de sagesse. Il a apparemment passé du temps en prison mais est très apprécié, respecté et écouté par la population. Des vendeuses, des travailleurs et des soldats viennent clore cet éventail hétéroclite de personnages.

Ce qui précède nous permet de parler des voix et des idéologies dans l'œuvre. Généralement, Besong utilise une multitude de perspectives dans ses œuvres, il présente ainsi de la même manière tous ses personnages - les opprimés et les oppresseurs - tels qu'ils sont, avec leurs qualités et leurs défauts, sans parti pris pour un camp ou pour un autre parce qu'il estime qu'ils sont tous corrompus. Cependant, dans Requiem for the Last Kaiser, l'approche est différente car l'œuvre est clairement orientée vers une idéologie socialiste comme pour donner un précepte moral à suivre. Il propose une solution possible à la fin de la dictature et prend ouvertement position pour les opprimés qui sont le seul espoir pour un changement 
social. Il adopte une sorte de manichéisme conscient en attribuant de bonnes caractéristiques à certains personnages (La Femme et Poet as Mandela) et de mauvaises caractéristiques à d'autres (le président Akhikikriki et ses gourous occidentaux).

Pierre Fandio (2011) porte un regard assez critique sur le choix des personnages de Besong. Il trouve que les portraits physiques et moraux de certains personnages fictifs sont trop proches de ceux de personnes existantes ou ayant réellement existé dans la société camerounaise et mondiale pour ne pas faire immédiatement penser à ces derniers. ${ }^{4}$ La critique de Fandio n'est pas complètement partagée dans cette étude. Étant donné le climat politique tendu au Cameroun à l'époque de l'écriture de la pièce de théâtre, pour quelles raisons Besong n'a-t-il pas fait preuve de plus de subtilité dans le choix et la description de ses personnages? Ce serait certainement pour affirmer sans détour ses convictions politiques et idéologiques et peut-être pour que les points de son message restent hardis et saillants dans l'esprit du lecteur/spectateur au lieu de les laisser s'évaporer dans les méandres de l'édulcoration littéraire.

Tout n'est pas foncièrement accusation, dénonciation et remise en question, plaidoyer et appel à l'action chez Besong. Il utilise des éléments humoristiques qui donnent à son théâtre un côté divertissant. La touche d'humour provient de la satire et du ridicule dont l'auteur couvre les personnages représentant les autorités. Il les présente comme des bouffons dont les mots et les actes peuvent difficilement être pris au sérieux et les apparentent à une plaisanterie. Cet humour singulier est essentiellement lié à la culture de l'auteur. C'est un humour caustique teinté d'ironie qu'il n'est pas toujours aisé de détecter car il est généralement créé par les situations et les paroles insensées liées à certains personnages.

L'auteur utilise également des références intertextuelles aussi vastes et diverses que la Bible et l'histoire mondiale sans restriction géographique ou temporelle, de l'Afrique du Sud au Japon en passant par les effets du communisme et la politique française et américaine en 
Afrique. Tout y passe, dans un ordre et une logique propres à l'auteur et qui animent l'imagination du lecteur. Les images et les symboles utilisés se dévoilent entre humour et références intertextuelles pour donner au texte toute sa saveur et évoquer chez le lecteur des sentiments tout à fait contradictoires et originaux. En tête de ces particularités se trouve la présence de plusieurs langues issues d'horizons divers dans le même texte et dans les mêmes prises de parole. Cette utilisation de la langue fait l'objet de la présente étude et constitue un moyen efficace de présenter les autres aspects de l'œuvre mentionnés préalablement.

Sarajeva (2011) explique que la première raison qui justifie le multilinguisme en littérature est la représentation réaliste de la situation linguistique d'un pays qui est favorisé par les migrations dues aux études, aux voyages, au travail, aux relations personnelles (mariages interethniques) et à l'exode rural. Les populations apprennent ainsi la langue de la région ou de la ville dans laquelle ils vivent, ou encore la langue de leurs amis et de leurs proches en plus de leur langue maternelle et d'une ou deux langues officielles. À cette complexité s'ajoute l'intercompréhension entre langues proches. Au Cameroun, la diversité géographique fait écho à la diversité linguistique. Le français et l'anglais en tant que langues officielles cohabitent avec plus de 200 langues nationales sur un territoire d'environ 475440 $\mathrm{km}^{2}$ (Mendo-Ze 1999: 46) occupé par approximativement 19,5 millions de personnes qui doivent y vivre en harmonie (données de la Banque mondiale 2010). L'utilisation de plusieurs langues dans l'œuvre montre donc la compréhension que l'auteur a de sa société.

Dans un univers culturel complexe où des langues différentes se côtoient, la cohabitation linguistique se présente sous diverses formes: emprunts, interférences, alternances, diglossie, polyglossie. Ces multiples formes de cohabitation se retrouvent dans Requiem for the Last Kaiser.

Il arrive aussi que la diversité identitaire se reflète dans les langues elles-mêmes, c'est le cas du pidgin-english. Le pidgin-english est la langue véhiculaire la plus répandue sur le 
plan communicatif au Cameroun et sert de lingua franca. Parmi les langues présentes dans l'œuvre, le français, le pidgin-english, l'éwondo, la langue spéciale de l'Ambassadeur et l'haoussa partagent le plus grand nombre d'occurrences. La langue spéciale est un mélange de jurons et d'expressions issus de l'argot américain. D'autres langues sont utilisées mais moins fréquemment, à savoir l'afrikaans et le zoulou, l'allemand, le latin et l'arabe. Toutes ces langues accompagnent l'anglais, langue dans laquelle la majorité de la pièce est articulée, sous forme d'alternance codique. Les personnages vont ainsi librement de l'anglais à l'une des langues mentionnées.

\section{Le public}

La réception de la pièce de théâtre, que ce soit de l'œuvre originale ou de la traduction, ne se fait pas sans heurts. Le public camerounais à qui est destiné la pièce originale partage le multilinguisme présent dans l'œuvre selon les critères présentés par Sarajeva (2001), ainsi même les Camerounais francophones pourront, a priori, comprendre le thème général et les différentes allusions mais la langue anglaise leur poserait encore un problème majeur. Pour les Camerounais anglophones c'est le style excentrique et compliqué de l'auteur et la gymnastique intellectuelle que requiert souvent sa compréhension qui serait une entrave à la compréhension de l'œuvre. La plupart des Camerounais pensent que Besong a commencé sa carrière en tant que dramaturge. En réalité, Besong est d'abord poète et le théâtre lui est venu bien plus tard. Cette précision est importante pour comprendre la relation qui lie l'auteur à son public et l'homogénéité de ses thèmes et de son style d'écriture. Le style de ses pièces de théâtre est empreint de langage poétique qui arrive à des moments les plus inattendus. Besong a décidé de quitter la poésie pour le théâtre pour des raisons qui concernent le public auquel il s'adresse. La situation sociopolitique explosive de cette époque au Cameroun demandait une 
réaction urgente qu'il se sentait le devoir de confronter. Babila Mutia nous confie comment Besong concevait son théâtre:

Drama that would be put on stage and watched by audiences across the nation became an urgent medium to express his ideas and transmit his message to a lethargic nation. Aware that Cameroonians do not read, he knew that if he opted to take the path of poetry he had begun in Nigeria, he feared, as he said, that he 'may die and remain unheard'. (Mutia 2007)

Cette citation renseigne sur le public cible de l'auteur qui est le peuple camerounais tout entier sans distinction linguistique apparente. Il est assez intrigant d'imaginer comment il espérait regrouper les factions linguistiquement opposées. La diversité linguistique y trouve certainement sa source. Une caractéristique du public camerounais apparente dans cette citation, est qu'il ne lit pas. Les thèmes, les motivations et les intentions de Besong ont un caractère universel qui n'exclut que les personnes qui se sentiraient directement attaquées par ses œuvres. Pourtant, son style représente l'élément le plus à même d'établir une distinction parmi les lecteurs/spectateurs.

Quant au public cible de la traduction, son hétérogénéité intrinsèque constitue le problème, de même qu'un certain manque de familiarité avec le théâtre africain. ${ }^{5}$ Le public cible de la traduction de Requiem for the Last Kaiser est le public francophone en général et le public francophone camerounais en particulier. Les francophones ainsi identifiés sont issus de cultures hétérogènes avec le français comme dénominateur commun. Ce français est parsemé de particularismes propres à chaque région ou à chaque pays. Selon Achille Mbembé (2007), la langue française n'est de nos jours plus la propriété exclusive de la France parce qu'elle est 'langue au pluriel'. Il semble évident qu'au-delà des différences régionales et 
nationales, il y a intercompréhension entre locuteurs de langue française. Pour la traduction de Requiem for the Last Kaiser il s'agissait donc d'utiliser une langue qui dise et qui raconte au monde la société décrite par l'auteur dans son œuvre et non une langue épurée qui nie l'identité individuelle pour s'adapter à l'idée du français d'une métropole qui n'existe plus et qui méprise la diversité. La langue joue ici un rôle crucial en raison du statut du français et de ses locuteurs qui ne promeuvent pas tous la diversité au même niveau.

Il est donc possible d'affirmer que le théâtre est la forme d'expression littéraire qui convient le mieux à une Afrique qui connaît encore dans bon nombre de pays une crise du livre et qui a besoin de s'ouvrir au monde pour transmettre, au-delà de son identité maintenant difficile à déterminer, son message à travers son expression linguistique. Besong considère la pièce de théâtre également comme une plateforme de conscientisation pour pousser la société en perdition au changement. La traduction apparaît alors comme un instrument de cette extériorisation car elle est par excellence ouverture à l'Autre. Cette ouverture à l'Autre comme nous l'avons vu est entravée par les difficultés liées à la traduction théâtrale, par le multilinguisme de la pièce de théâtre Requiem for the Last Kaiser et par l'hétérogénéité du public auquel est destiné sa traduction. Ce public n'est pas toujours habitué au théâtre africain et n'aspire pas toujours à la diversité linguistique. Pour trouver un terrain d'entente qui permet la traduction, il fallait s'assurer de la fonction réelle du multilinguisme dans la pièce pour savoir si elle précède ou non le message de l'auteur et si elle doit être conservée ou supprimée. Il a donc été nécessaire d'ajouter au travail théorique déjà effectué, un travail sur le texte.

\section{Méthode effectivement adoptée pour la traduction}

Ayant identifié les enjeux théoriques liés à la traduction théâtrale et à la traduction multilingue ainsi que les stratégies de traduction suggérées et connaissant la spécificité de 
Requiem for the Last Kaiser, son auteur et le contexte socioculturel camerounais, l'étape suivante a consisté à appliquer les informations ainsi recueillies à la traduction de cette œuvre qui regroupe à la fois théâtre et multilinguisme. Dans cette dernière partie, nous allons aborder les défis rencontrés et expliquer les différentes décisions traductionnelles qui ont été prises.

Le premier défi était de décider s'il était réellement nécessaire de traduire une telle œuvre. En effet, si on considère les nouvelles théories de la traduction théâtrale, le jeu des acteurs sur la scène peut permettre de résoudre les problèmes d'ambigüités, rendant quelque peu caduc le besoin de traduire. Cependant, cela ne remet pas en cause la validité de la traduction de l'œuvre, étant donné que la langue principale - l'anglais - représente environ soixante-dix pourcent de la pièce. De plus, les traductions de textes multilingues qui ont déjà été effectuées attestent de la possibilité de traduire Requiem for the Last Kaiser. Il en découle la nécessité de déterminer comment traduire la pièce de théâtre, notamment en ce qui concerne l'hétérogénéité linguistique.

En l'absence de l'auteur, qui est décédé, il est difficile d'établir son intention d'écriture et de savoir quelle importance et quelle fonction il attribuait au multilinguisme. En théorie, il faut conserver le multilinguisme pour des raisons idéologiques. En effet, le multilinguisme est utilisé en littérature postcoloniale pour s'opposer à la domination des langues coloniales et en réaction aux conventions d'écriture monolingues préconisées pour des raisons financières et pour faciliter leur réception par le public cible. Ce choix gomme la multiplicité linguistique qui est la caractéristique même du texte source. Un auteur peut également avoir plusieurs raisons d'intégrer différents éléments linguistiques dans ses textes. À titre d'exemple, de nombreux théoriciens pensent que les écrivains incluent le multilinguisme dans leurs œuvres pour faire un portrait réaliste de la société ou de la communauté qu'ils décrivent ou pour marquer la nationalité d'un personnage. La véritable 
difficulté est que nous ne comprenons pas entièrement les textes, multilingues ou non, traduits ou non (Meylaerts 2010). La voie vers une compréhension réelle des textes commence en mettant l'accent sur la fonction spécifique que l'auteur a assignée à l'utilisation de la langue ou des langues pour exprimer ses idées. Pour orienter la traduction, la théorie du Skopos a été utilisée afin d'identifier l'intention d'écriture de Besong et la fonction du multilinguisme dans l'œuvre. Il a ainsi été décidé de conserver la diversité linguistique car il semble que la langue est utilisée pour servir l'objectif d'expression et de communication de chaque personnage comme si chacun d'entre eux avait l'opportunité et la possibilité de parler naturellement et librement sans aucune contrainte ou intention délibérée de résistance à l'anglais. Par conséquent, l'idée de résistance à l'hégémonie d'une langue dominante ne semble pas être la raison principale de l'utilisation du multilinguisme dans la pièce de théâtre comme le montrent les langues en présence. Ceci étant dit, la question demeure de savoir comment traduire, quelle stratégie générale adopter, comment équilibrer étrangeté et familiarité, à quelles langues faire correspondre les langues du texte source et quelles procédés de traduction utiliser pour les portions de texte contenant ces éléments multilingues.

La première étape de travail sur le texte a consisté à relever toutes les occurrences multilingues qui ont ensuite été classées en monologues et en dialogues. Celles-ci ont été analysées, traduites et commentées. L'étude du contexte d'existence de la traduction multilingue montre que généralement le choix de stratégie de traduction ne dépend pas du texte original. La traduction partielle de Requiem for the Last Kaiser n'avait pas de commanditaire car elle a été effectuée dans le cadre d'une recherche universitaire. Cependant, la traduction, bien qu'elle conserve le multilinguisme du texte source préconisé par la traduction multilingue, n'a pas été orientée vers une approche sourcière. En effet, malgré les conclusions établies après l'analyse des conventions théoriques et pratiques, la traduction n'a pas toujours suivi les recommandations suggérées car face au texte à traduire les 
considérations théoriques ont en quelque sorte perdu de leur force. Le texte source a ses règles et que celles-ci ne s'appliquent pas toujours au texte cible. Il fallait donc, réplique après réplique, personnage après personnage, prendre du recul, identifier la fonction des prises de parole multilingues, leur lien et leur dynamique avec les autres éléments de la pièce de théâtre et décider soit d'atténuer l'étrangeté du multilinguisme, soit de la supprimer ou d'allier étrangeté et familiarité en utilisant à différentes doses les procédés énumérés précédemment. Il a souvent fallu ajouter des références étrangères au texte source mais indispensables au texte cible. En fin de compte, il fallait que le texte cible ne soit pas complètement transparent mais que le metteur en scène puisse trouver des repères pour le jeu des acteurs permettant au public de suivre la pièce car même le texte source en-soi n'est pas facile à comprendre. Nous avons utilisé le littéralisme, l'adaptation et l'étoffement ainsi que d'autres procédés nous permettant d'obtenir l'effet souhaité chez le public.

Pour ce qui est des langues utilisées pour la traduction, les décisions n'ont pas été prises unilatéralement en opérant une généralisation pour chaque langue. Les langues dans le texte source sont le français standard et ses différents registres, le camfranglais, le pidginenglish, une adaptation du franglais pour la langue spéciale de l'Ambassadeur américain, l'anglais, l'éwondo, le zulu, l'afrikaans, l'allemand, le latin et l'arabe. Nous avons opté pour le français comme langue principale correspondant à l'anglais de l'œuvre originale. Lorsqu'il s'agissait d'un 'mauvais' anglais, nous l'avons traduit en utilisant le camfranglais principalement pour le personnage État-Major dont la langue a pour fonction de s'adapter à son contexte d'élocution. Parfois, il a été nécessaire de conserver l'anglais dans le texte cible dans le but de maintenir la logique du texte original en respectant les caractéristiques des personnages. Le français dans le texte source est resté tel quel et caractérisé par le manque de correction linguistique pour le président Akhikikriki, le mélange de registres, et dans certains cas, il a été traduit en anglais pour servir l'objectif d'expression du personnage. Le pidgin- 
english a été traduit en camfranglais la plupart du temps, il a été conservé dans les dictons et les proverbes et adjoint d'une explication littérale ou d'une gestuelle. Le hausa a été traduit en éwondo, souvent laissé tel quel ou accompagné d'une gestuelle explicative. Le terme spartiate «bouai» est remplacé par un terme en ancien français car il n'était pas indispensable à la transmission de l'intention de l'auteur, ni à la réception du message par le public cible.

\section{Exemples d'analyse, de traduction et de commentaire des prises de parole multilingues}

À titre d'exemple, un extrait de Requiem for the Last Kaiser a été analysé pour démontrer le processus de traduction en fournissant les fondements des prises de décision sur fonds des stratégies de traduction qui ont permis d'obtenir un texte cible.

\begin{tabular}{|l|l|}
\hline \multicolumn{1}{|c|}{ Texte source } & \multicolumn{1}{|c|}{ Traduction du texte source } \\
\hline Voice from coffin: & VOIX VENANT DU CERCUEIL \\
Are you there? ... The people understand me, very & Vous m'entendez ? ... Le peuple me comprend très bien. \\
well. I also understand them. We know what we & Je comprends aussi le peuple. Nous savons ce que nous \\
want (pause). At the Great Gathering, I'll & voulons (pause). Pendant la Conférence nationale \\
mesmerize them: Soweto, Falling Cocoa prices in & souveraine, je vais les éblouir: Soweto, la chute des prix \\
the World Market, that stone country Butcher dog & du cacao sur le marché mondial, le pays aride de ce \\
Botha. By decree number one million and ninety- & chien boucher, Botha. Par décret numéro un million \\
ten ... ninety-ten (pause). I will get them where I & quatre vingt-dix dix ... quatre-vingt-dix dix (pause). Ils \\
want. (a spitting noise). Take these journalists off & feront ce que je voudrai (bruit de crachat). Arrêtez ces \\
and torture them. I am the Consciousness, the & journalistes et torturez-les. Je suis la Conscience, le \\
Tempo and Heart-Throb of Iduote. The He-alone & Tempo et la Coqueluche d'Iduote. Le Seul, le Guide. \\
and Guide. (short pause). I am the One-Man-Band & (petite pause). Je suis l'Homme-Orchestre... Je suis le \\
\hline
\end{tabular}


... I am the Universal Pedagogue and Pointing Rod. I'm in all places at the same time. (to an imaginary group). Je vous ai compris. I'll tax them for the air I provide... (short pause). In this Total Coming, I will be the Alpha and Omega, the last and only pharaoh in paradise. The history of the historicity of Agidigidi. (with a stage whisper). Tax them for the air I provide ... and they know that I can be tough... By decree number one million and ninetyten... yes... one million and ninety-ten (with a hoarse whisper). I'll be in politics till I die... I give small bouai power... one small bouai power... Shege dan banzaar... I go come dey (a long pause). Amot, Ma'a ding Sonara money... Oweh, money mbeng. Wa ding money? Bebele zamba-a!

Coffin opens and reveals the cadaverous face of His Royal Majesty Akhikikriki 'the First, the Last' for a moment. (pause) La tricherie, la demagogie, la traguerie ... all mixed together ... le vandalisme ...

Coffin closes entombing His Majesty Baal Akhikikriki Njunghu, the First and the Last Miracle for the night

Violent but choked snoring accompanied by funerary music... (voice as if in transition). Wa-
Pédagogue universel et le Bâton de commandement. Je suis omniprésent. (à un groupe imaginaire). J'en suis conscient. Je vais leur taxer l'air que je pourvois... (petite pause). Je serai l'Alpha et l'Omega, le Dernier et le Seul pharaon au paradis. L'histoire de l'historicité d'Agidigidi. (il chuchote, sa voix est rauque). Taxez l'air que je pourvois... et ils le savent, je peux être coriace... Par décret numéro un million quatre-vingt-dix dix ... oui, je dis bien... quatre-vingt-dix dix. (d'une voix basse et rauque). Je resterai dans la politique jusqu'à ma mort... Moi, je distribue de petits pouvoirs à la Confrérie des Conards ... un petit peu de pouvoir à la Confrérie des Conards ... Shege dan banzaar... Espèces de Bâtards... j'arrive, I go come dey (une longue pause). Amot, Ma'a ding Sonara money... Oweh, money mbeng. Oui j'aime l'argent du pétrole... L'argent c'est bon. Wa ding money? Toi aussi tu aimes l'argent? La vérité devant Dieu. Bebele zamba-a!

Le cercueil s'ouvre pour dévoiler pendant un instant le visage au teint cadavérique de sa Majesté royale Akhikikriki «Premier et Dernier». (pause). La tricherie, la démagogie, la traguerie ... tout ça mélangé ... le vandalisme ...

Le cercueil se referme, ensevelissant pour la nuit, sa 


\begin{tabular}{|l|l|l|}
\hline $\begin{array}{l}\text { ding money? ... M'a ding Sonara money...Sonara } \\
\text { money abui... Je vous ai compris... Za-ah bi ké... }\end{array}$ & $\begin{array}{l}\text { Majesté Baal Akhikikriki Njunghu, le Premier et le } \\
\text { Wa Ke vé? }\end{array}$ \\
(Besong 1998:1) & $\begin{array}{l}\text { Des ronflements violents mais étranglés accompagnent } \\
\text { une musique funèbre... (voix comme en transition) Wa } \\
\text { ding money ? M'a ding Sonara money... Sonara money } \\
\text { abui... Tu aimes l'argent ? Moi, j'aime l'argent du } \\
\text { pétrole, l'argent du pétrole est beaucoup. J'en suis } \\
\text { conscient ... Za-ah bi ké... Wa Ke vé? Viens, on s'en } \\
\text { va. Mais tu vas où ? }\end{array}$
\end{tabular}

Requiem for the Last Kaiser s'ouvre donc sur un monologue du président Akhikikriki qui commence par une question qui fait penser qu'il recherche un vis-à-vis, il interpelle le public pour signaler que la pièce a commencé et qu'il a besoin de son attention. 'Are you there ?' (Besong 1998: 1), première phrase du texte, peut être interprétée comme une interrogation pour se rassurer qu'il n'est pas seul, qu'il a de l'importance ou qu'il ne passe pas inaperçu. C'est un moyen de se mettre en relation à l'autre qui est également utilisé dans les phrases suivantes: 'The people understand me, very well. I also understand them. We know what we want' (Besong 1998: 1). On suppose immédiatement que le personnage a une relation très étroite avec son peuple parce qu'ils se comprennent mutuellement. Ainsi, 'the people' est lié à 'me' dans la deuxième phrase, 'I' est associé à 'them' dans la troisième phrase et suivi d'un 'we' dans la phrase suivante.

La scission arrive avec le changement des pronoms personnels qui deviennent subitement 'I' et qui ne changeront plus jusqu'à la fin du monologue: 'I'll mesmerize them [...] I will get them where I want. [...] I am the Consciousness, the Tempo and Heart-Throb 
of Iduote. The He-alone and Guide (short pause). I am the One-Man-Band. I am the Universal Pedagogue and Pointing Rod. I'm in all places at the same time' (Besong 1998: 1). Le rapport à l'autre disparait. Ce changement montre les véritables facteurs qui conditionnent l'intercompréhension du président avec le peuple; c'est la mégalomanie et l'égocentrisme du personnage qui manipule le peuple à sa guise. L'intercompréhension est factice. Le rythme du texte est saccadé, entrecoupé par les multiples pauses du personnage; comme s'il admirait son portrait et devait rechercher les mots adéquats pour se décrire. Le tempo est créé par son autonomisation, il n'y a pas mieux que lui, il est premier de tout et de n'importe quoi; ceci souligne le ridicule de la situation. Il est 'le Seul' ('He-Alone') au-dessus de tout et de tous. Le choix du monologue en tant que structure répond bien au narcissisme du personnage car il permet de révéler les sentiments du personnage et répond à la fonction introspective, délibérative pour une analyse des actions passées du personnage et une préméditation des actions qu'il compte effectuer.

Le monologue a une signification qui évoque souvent, en plus de l'introspection, la démence ou la folie. Cette première prise de parole nous permet de caractériser le personnage physiquement mais surtout moralement comme un personnage chargé de contradictions dont la politique est basée sur le nombrilisme, les faux-semblants et l'instrumentalisation du peuple. Son nom est une source d'humour et de ridicule; l'assonance d'Akhikikriki fait penser à un criquet. Ce n'est pas la bête la plus noble du règne animal, ce qui est incompatible avec l'idée d'un président qui a une fonction noble et importante. Son cri est également très caractéristique, strident, peu agréable à écouter, cela nous rappelle le verbiage d'Akhikikriki.

La compétence linguistique du président est l'élément qui porte le plus à réflexion. Il semble ainsi être parti dans un délire où les langues se mêlent. Dans ce monologue, il utilise le français et l'anglais de manière incorrecte: 'By decree number one million and ninety-ten 
... ninety-ten [...] La tricherie, la demagogie, la traguerie' (Besong 1998: 1). L'éwondo, l'haoussa, le pidgin-english ainsi qu'un terme spartiate 'bouai' s'ajoute à son récital linguistique. 'Je vous ai compris' (en français dans le texte) se réfère tout d'abord à un discours du Général De Gaulle en Algérie, mal interprété par les deux camps pendant la guerre. On pensait qu'il allait protéger les droits des pieds-noirs et donner son indépendance à l'Algérie. C'est aussi une phrase qui se réfère certainement à une phrase connue ('J'en suis conscient') prononcée par le président du Cameroun à l'occasion d'un discours à la nation. Lorsque le pays connaissait les premières répercussions de la crise économique, le président prononça cette phrase pour montrer sa solidarité envers son peuple. ${ }^{6}$ Mais elle fut interprétée par le peuple comme de la condescendance car n'étant pas suivie de mesures concrètes de redressement économique. 'Bouai' est un terme utilisé pour désigner une confrérie de Sparte à laquelle les jeunes hommes adhèrent dès l'âge de sept ans. L'utilisation de 'small Bouai power' dans le texte rappelle la segmentation ethnique et clanique du pouvoir au Cameroun. Akhikikriki daigne ainsi accorder aux citoyens divisés par ethnies un certain pouvoir en fonction de leur regroupement afin de garantir son hégémonie, surtout pour bien montrer qu'il détient le pouvoir et peut le donner à qui il veut. Il semble utiliser les autres langues du texte pour s'adresser aux différentes ethnies dans des langues qu'ils peuvent comprendre.

Le pidgin-english est représenté dans ce monologue par la phrase 'I go come dey' (Traduction littérale: I will come there) suivie d'une longue pause. Le pidgin-english a des usages militants qui s'attachent à son utilisation; celle-ci est liée à la période des agitations urbaines des années 1990 au Cameroun. Les membres de l'opposition avaient choisi pour fief la capitale économique, Douala, et avaient coutume de défier le président de quitter la capitale politique, Yaoundé, pour venir à leur rencontre. 'I go come dey' dans le texte fait certainement référence à une promesse de briser la rébellion. ${ }^{7}$ 
Après une longue pause, le président exprime le fond de sa pensée et les raisons qui résument son action. Il ne s'agit pas de communier avec le peuple et encore moins d'être ferme dans le but de faire avancer le pays ou d'éclairer de ses lumières le bas-peuple. La motivation d'Akhikikriki c'est l'accumulation des revenus monétaires, autrement dit l'argent que rapporte l'exploitation pétrolière par la SONARA (Société nationale de raffinerie). Il s'exprime alors en éwondo (qui est également la langue du président du Cameroun). Il prend le public à témoin de manière familière en disant 'Amot' (littéralement, c'est une interpellation généralement adressée à un homme, par extension on entend souvent dire: 'mon frère...') et lui demande s'il partage sa cupidité et son avidité, et le jure devant Dieu.

L'intonation est très importante pour transmettre le passage car on ne voit pas le personnage, son visage n'apparaît qu'un court instant; par conséquent, on ne peut pas compter sur les gestes pour compléter la voix. La tonalité de cette prise de parole est d'abord dramatique et doucereuse. Akhikikriki semble même dire avec douceur qu'il faut torturer les journalistes. Ensuite, le ton se veut affirmatif et condescendant; Akhikikriki s'impose comme la solution à tout mal. Le ton devient enfin lyrique, le personnage nous fait partager ses sentiments intimes. L'inconsistance du personnage relève presque du délire puisqu'il parle dans son sommeil. Il appelle l'argent vers lui, comme si cet argent lui échappait: 'mais où vas-tu ? Viens ici...'. Devrait-on croire à une prémonition ? Certainement ! Avec le cercueil, le teint cadavérique du personnage et le fait de dormir dans le cercueil, le décor est planté. En conclusion, l'objectif de ce monologue est de susciter le rire face au ridicule d'une personne qui dort dans un cercueil, qui a l'air d'avoir un pied dans la tombe et qui divague en se prenant pour le nombril du monde. Le multilinguisme ici a pour fonction selon la liste d'András Horn (1981) de produire un effet comique en général.

\section{Commentaire de la traduction}


En général, la traduction a gardé un tempo naturel, l'attention a été portée sur les contraintes sonores et la syntaxe pour que le texte soit plausible et convaincant en français. Par conséquent, le littéralisme n'a pas toujours été préconisé mais il s'est agi de donner au texte une dynamique propre inspirée du texte original. Les sons, la musique, la simplicité et le mélange de registre ont été privilégiés pour que le texte soit acceptable sans trahir l'intention de l'auteur.

Pour les parties déjà en français dans le texte source, la phrase 'Je vous ai compris', dans la traduction, pouvait rester telle quelle, être traduite directement en anglais 'I've understood you' ou être transformée en la remplaçant par sa connotation connue au Cameroun 'J'en suis conscient'. La traductrice a opté pour la troisième possibilité. ' 'Je vous ai compris' en allusion à la phrase du Général De Gaulle aurait bien fonctionné mais l'allusion au 'Bouai' rapproche davantage du contexte camerounais. L'anglais 'I've understood you' aurait pu créer un effet volte-face par rapport à l'original c'est-à-dire que le président qui est francophone parle peu l'anglais, pourtant dans Requiem for the Last Kaiser, il s'exprime couramment en anglais et son français est truffé de fautes. Traduire en anglais les morceaux de phrases en français dans le texte source aurait replacé le texte dans une sorte de réalité, mais les spectateurs et les lecteurs du texte cible ne connaîtront pas l'original, ce qui fait perdre tout effet. 'J'en suis conscient' par contre, apporte un élément de surprise à cause de son étrangeté à ce moment du discours. Sans se fondre complètement dans le texte, il contribue à souligner l'incohérence et le narcissisme du président. Le spectateur se demande tout de suite si le personnage a tous ses sens ou si c'est lui qui comprend mal. On peut reprocher à ce choix de manquer d'un pronom personnel défini comme dans l'original 'Je vous ai compris', mais la phrase suivante montre que le personnage ne s'adresse en réalité à personne: le président est toujours devant son reflet narcissique. Avec 'J'en suis conscient' il n'y a aucun doute que le sujet de ses réflexions n'est nul autre que lui. 
Le segment ' La tricherie, la démagogie...' etc. en français dans l'original a été laissé tel quel dans la traduction car c'est une énumération des maux dont souffre la nation et pour l'auteur, cette tare est, d'après les anglophones, surtout le fait des francophones. Traduire en anglais équivaudrait à dénaturer l'intention de l'auteur. De plus, les sonorités (terminaisons en -ie) sont plus importantes que le sens.

Le mot spartiate 'Bouai' pose un problème de traduction singulier. Au départ, la traductrice a pensé ne pas traduire le mot car le contexte peut aider à comprendre que le président omniprésent et omnipotent distribue le pouvoir avec parcimonie à un groupe de personnes qu'il traite ensuite de bâtards. Par la suite, étant donné qu'il y a une certaine liberté dans le choix des références culturelles du fait que l'auteur en fait usage sans restrictions, il était possible de se servir de cette allusion pour introduire un élément d'humour. Au XVe siècle à Rouen en France, il existait une Confrérie de joyeux lurons appelés les 'Conards' officiellement autorisés par le Parlement à se moquer de tous et de tout à l'exception du Roi ${ }^{9}$. Le public pourra ainsi en rire même s'il ne sait pas exactement de quoi il s'agit. La Confrérie des Conards a aussi l'avantage d'apporter une touche d'ironie à la pièce pour contribuer à l'originalité de la traduction. Elle apporte du rythme à la réplique grâce à la répétition de Confrérie des Conards et à la répétition du son '-ard' avec bâtards.

L'éwondo, le pidgin-english et l'haoussa ont été gardés dans le texte traduit mais ont les équivalents en français y sont ajoutés soit avant, soit après selon que le rythme s'y prête. L'élément de confusion a ainsi été accentué pour faire passer le message. Le texte en français est en fin de compte plus saccadé et plus étrange que son original, ce qui contraste avec la simplicité du langage en général que vient perturber le mélange de registre. L'emphase a été introduite et les éléments répétés, changés pour accentuer l'égocentrisme et la cupidité du personnage: 
Moi j'aime l'argent du pétrole, l'argent du pétrole est beaucoup.

Ma ding Sonara money, Sonara money abui. (Besong 1998: 1)

J'aime l'argent de la Sonara, l'argent de la Sonara est beaucoup. (traduction directe)

Le sigle Sonara a été supprimé dans la traduction en français parce qu'il apparaît déjà dans la phrase en éwondo à laquelle elle est juxtaposée. Cette répétition aurait alourdi le texte traduit.

Akhikikriki termine son discours éveillé avec la phrase 'Bebele zamba !' C'est une expression que l'on utilise couramment au Cameroun et signifie littéralement 'Vraiment de Dieu', mais elle n'aura pas de sens pour d'autres francophones. La traduction choisie est 'La vérité devant Dieu' au lieu de 'Je le jure devant Dieu', qui aurait été trop transparent.

'I go come dey' reste dans le texte et est traduit par 'j'arrive' (qui est moins formel que j'y arrive) et sonne comme une menace adressée aux bâtards. Dans la version française, il semble qu'il arrivera plus vite que dans le texte source, c'est une perte mais c'est surtout pour éviter le ralentissement du rythme causé par la juxtaposition des deux phrases en français et en pidgin-english. 'Shege dan banzaar' est une injure en haoussa qui signifie bâtards en français. La phrase a été étoffée avec 'Espèces de bâtards' pour donner plus de poids à l'injure.

Les multiples pauses permettent d'assurer les transitions entre les différentes langues et peuvent aussi faciliter la compréhension du message. Le plus difficile dans ce monologue était d'arriver à recréer le comique et le ridicule qui sont essentiels à cette première partie, sans donner davantage d'explications qui auraient rendu le texte trop transparent au public/lecteur. Le texte source ne s'est pas toujours laissé traduire littéralement même si ce n'est pas la logique qui y prime. Il a donc été préférable d'assurer l'harmonie des sonorités et la transmission des effets, ce qui explique quelques écarts structurels entre le texte source et le texte cible. 
La traduction littérale a été utilisée en ce qui concerne les dictons et les proverbes entre autres. La non-traduction, le calque et l'emprunt ont été choisis dans les cas où les références culturelles et les éléments multilingues étaient porteurs de sens en-soi.

Chop a chop, monkey pass monkey... Awoof no get bone. (Besong 1998: 41)

[You eat, I eat, beyond a monkey is a monkey... Freebie doesn't have bone] (traduction directe)

Vous mangez, je mange aussi. Un singe ne dépasse pas un autre singe... le gratis n'a pas l'os.

Dans la traduction, le dicton en pidgin-english a été supprimé et remplacé par une traduction littérale en français. Le registre est familier car il s'agit d'une vendeuse. Il n'y a pas d'explication supplémentaire parce que c'est le contexte qui fait comprendre le dicton. La traduction littérale apporte assez d'étrangeté et conserve bien la fonction originelle des dictons et des proverbes. Néanmoins dans cette réplique d'un travailleur, il a été question de garder le dicton en pidgin-english et de l'accompagner d'un langage gestuel approprié.

He is the intellectual who couldn't pawn his soul like the rest (in pidgin English). Wetin my eyes dey see, my mouth no fit talk-am o! And that by Presidential decree. (Besong 1998: 27)

C'est l'intellectuel qui a refusé de vendre son âme comme tout le reste (en pidginenglish). Wetin my eyes dey see, my mouth no fit talk-am o! (Il accompagne ses paroles d'un geste de la main pour se couvrir les yeux et ensuite la bouche) Et ça c'est par décret présidentiel oh !

Ce que mes yeux voient, ma bouche ne peut pas parler oh! (Traduction directe) 
Un autre exemple de traduction où nous avons choisi de transmettre le message en français et en camfranglais pour remplacer l'anglais standard et le pidgin-english de l'original est tiré du monologue d'Abessolo - la main armée du pouvoir. 'Himihimi' en hausa a été remplacé par 'aloil' qu'on utilise pour désigner les parties génitales de la femme en béti (l'éwondo fait partie des langues béti); nous y avons ajouté une didascalie qui par un geste rapide montre l'action de couper une partie inférieure du corps. C'est un exemple de traduction entre deux langues africaines afin de garder l'illusion de la réalité parce qu'Abossolo comme son nom l'indique est certainement originaire d'une des tribus béti.

We went to one joint ... fine fine girls are there. And there was that man whose wife with a sharp matchet I cut her himihimi. There, the fool was looking at me in that strange way...

Nous sommes allés dans un bar... il y avait plein de belles petites. Et il y avait cet homme, avec une machette bien tranchante j'avais coupé l'aloil de sa femme (il accompagne ses mots d'un geste rapide de la main pour montrer l'action). L'imbécile me regardait bizarre...

L'étoffement a été utilisé comme un moyen de clarification sémantique ou pour créer l'effet voulu chez le public. La juxtaposition de l'élément multilingue et son explication a servi à assurer sa compréhension effective. La gestuelle s'est également avérée essentielle pour compléter le sens entre le dit et le non-dit dans le texte. L'analyse spécifique de ces prises de parole a ainsi permis d'approfondir la compréhension du texte tout entier et a facilité la traduction. Les commentaires des traductions proposées permettront d'éclairer une éventuelle traduction complète de l'œuvre. 
Pour la traduction de Requiem for the Last Kaiser il est vrai que l'approche choisie de clarifier le texte cible pour essayer de recréer l'effet produit par le texte source aurait dû être laissé aux soins du metteur en scène. À la limite, il aurait suffi d'ajouter des didascalies plus précises que cette du texte source dans le but de faciliter la tâche du metteur en scène pour rester fidèle aux recommandations théoriques et pratiques. Mais il semblait important de prendre en considération l'immédiateté du texte théâtral qui implique que les spectateurs présents doivent saisir le sens du dialogue immédiatement (Zatlin 2005: 1) c'est-à-dire aussitôt qu'il est prononcé en une seule fois; contrairement aux textes lus, le texte dramatique oral n'a pas de note en bas de page. La traductrice a pris le risque de diluer le multilinguisme de l'œuvre pour faciliter la compréhension du public cible. Le texte traduit peut ainsi paraître trop transparent conformément aux présupposés idéologiques autour de l'hégémonie des cultures dominantes en traduction. Il n'en demeure pas moins que le traducteur doive faire des choix conscients ou inconscients. Le vieux débat sur l'opposition entre forme et contenu retrouve ici sa place. Le public cible ne peut pas être complètement ignoré. Tout revient à déterminer pour quelle raison la traduction est-elle effectuée. La traduction devrait permettre un échange, et pour qu'il y ait échange, il faut qu'il y ait ouverture et acceptation de l'Autre. C'est la raison pour laquelle nous avons adopté une forme de traduction hybride, qui montre le côté étrange de l'œuvre originale mais sans laisser le récepteur dans le noir. La traduction offre ainsi au metteur en scène des possibilités pour orienter ses choix finaux.

\section{Conclusion}

Un traducteur complet devrait traduire et connaître les mécanismes qui constituent l'acte de traduire en référence au cadre théorique qui entoure l'œuvre et sa traduction. La traduction théâtrale se réalise en gardant à l'esprit que la pièce obtenue est destinée à être représentée. Cependant, le traducteur ne peut pas prendre en charge tous les aspects inhérents au texte 
théâtral y compris son caractère immédiat et jouable. Dans ce contexte, les choix traductionnels doivent conserver les caractéristiques intrinsèques au texte de départ. Cette prise en compte assure la participation des autres acteurs du milieu théâtral et la réception ultérieure du texte par le public cible. La diversité linguistique et culturelle inhérente à la pièce de théâtre Requiem for the Last Kaiser ajoute un facteur supplémentaire à la traduction car elle apporte un caractère hétérogène qui rompt avec l'unité relative d'un texte littéraire.

Un texte multilingue est avant tout un texte comme un autre ayant un but - ce but peut être de n'avoir aucun but - et des caractéristiques qui lui sont propres. La diversité linguistique du texte augmente la complexité de l'analyse mais n'est pas un frein à la compréhension ou à la communication. Requiem for the Last Kaiser nous a permis de comprendre de manière concrète les limites de l'application unilatérale des théories souvent prescriptives à la traduction des œuvres littéraires. Le traducteur doit aller chercher le sens du texte là où il se trouve, il doit être à l'écoute du texte source. Nous avons vu que très souvent l'auteur n'essaie pas de faire du sens comme c'est le cas dans certaines parties des monologues. D'où l'importance de suivre les fluctuations du texte pour le comprendre convenablement plutôt que de lui imposer des règles à suivre. À notre avis, on ne peut pas déterminer la forme et le contenu du texte selon la théorie avant la traduction effective sans courir le risque de se tromper et de produire un texte inadéquat. La théorie offre une sorte d'éventail de possibilités dans lequel le traducteur peut venir puiser en fonction des besoins dictés par le texte à traduire. Les deux traductrices de romans multilingues, Ramiere et von Flotow, ont opté pour le calque, l'emprunt et la traduction littérale pour rendre le caractère multilingue des romans originaux; cependant, nous avons eu besoin de procédés supplémentaires pour la traduction de Requiem for the Last Kaiser. En d'autres termes, nous avons vérifié que ces mots, expressions et phrases multilingues n'étaient pas en-soi porteurs de sens. Au quel cas, nous avons décidé en fonction du potentiel de traitement cognitif du 
public cible et du rythme du texte cible de laisser les éléments multilingues tels quels, de leur adjoindre une explication succincte si nécessaire, de les supprimer ou simplement d'en transmettre uniquement le sens en délaissant la forme.

Il n'a certes pas été possible dans l'étendue de cette étude de présenter tous les exemples de solutions traductionnelles et d'attester entièrement avec des preuves à l'appui, de l'efficacité de la traduction pour le public cible, mais en prenant en considération les caractéristiques du langage dramatique, nous avons essayé d'élaborer un texte que le travail du metteur en scène et des acteurs peut compléter car la traduction ainsi effectuée permet de faire un choix ultérieur et conscient soit de garder étrangeté et familiarité, soit de choisir l'un ou l'autre. De plus, la traduction est partielle mais elle est généralisable à toute l'œuvre car elle s'inspire de son analyse globale. Il n'en demeure pas moins que ce sont des choix et des préférences qu'il faudra nécessairement faire valider par le public. Dans cette étude, il s'agissait surtout de présenter une œuvre littéraire exceptionnelle et de susciter le débat et de l'intérêt pour la traduction d'une écriture théâtrale multilingue qui, dans Requiem for the Last Kaiser exprime d'une part la liberté et l'identité trouble du monde globalisé et d'autre part se situe au-delà de la division linguistique de la littérature camerounaise et contribue à prouver qu'elle existe, qu'elle doit être promue et rendue accessible grâce à la traduction.

\section{Références}

Ambanasom, Shadrach Ateke (2000), 'Cameroonian and Kenyan writers in Politics: An analysis of the Works of six Playwrights', Observatoire réunionais des arts, des civilisations et des littératures dans leur environnement, http://laboratoires.univreunion.fr/oracle/documents/400.html. Consulté le 27 novembre 2011. 
Bandia, Paul Fadio (2008), Translation as Reparation: Writing and Translation in Postcolonial Africa, London: Routledge.

Bassnett, Susan (1998), 'Still trapped in the labyrinth: further reflection on translation and theatre', in S. Bassnett and A. Lefevere (dir.), Constructing Cultures: Essays in Literary Translation, Clevedon: Multilingual Matters.

(2008), Translation Studies, London: Routledge.

Batchelor Kathryn (2009), Decolonizing Translation: Francophone African Novels in English Translation, London: Routledge.

Besong, Bate (1998), Requiem for the Last Kaiser: A Drama of Conscientisation and Revolution, Limbe: Press Book.

Diawara, Gaoussou (2001), 'Le théâtre africain à la croisée des chemins: pour un développement dans l'équilibre', http://www.temoust.org/le-theatre-africain-a-la croisee,14346. Consulté le 15 septembre 2011.

Fandio, Pierre (2011), 'La littérature camerounaise d'expression anglaise: heurs et malheurs d'un champ culturel en constitution', Mondes francophones, http://mondesfrancophones.com/espaces/afriques/\%C2\% AB-la-litterature-camerounaised\%E2\%80\%99expression-anglaise-heurts-et-malheurs-d\%E2\%80\%99un-champ-culturel-enconstitution-\%C2\%Bate Besong. Consulté le 15 juin 2011. 
Horn, András (1981), ‘Ästhetische funktionen der sprachmischung in der literatur’. Arcadia, $16: 3$, pp. 225-241.

Mackey, William (1993), 'Literary diglossia: bilingualism and cosmopolitanism in literature'. Visible language, 21, pp. 40-66, http://visiblelanguagejournal.com/web/abstracts/abstract/literary_diglossia_biculturalism_and _cosmopolitanism_in_literature. Consulté le 17 juin 2012

Mbembé, Achille (2007), 'Francophonie et politique du monde', Congopage Blog, http://www.congopage.com/Achille-MBEMBE-Francophonie-et. Consulté le 09 juillet 2012.

Mendo-Ze, Gervais (1999), 'Contextes du français au Cameroun', in G. Mendo-Ze (dir.), Le français langue africaine: enjeux et atouts pour la francophonie, Paris: Plubisud.

Meylaerts, Reine, (2010), 'Multilingualism and translation', in Y. Gambier, \& L. van Doorslaer (eds.), Handbook of translation studies. Amsterdam: John Benjamins.

Mutia, Babila (2007), 'Besong: The man, his ideas, his vision and his life', http://www.batebesong.com/2007/04/besong_the_man_.html. Consulté le 09 décembre 2012.

Ricard, Alain (2004), The Languages and Literatures of Africa, Oxford: James Currey.

Ricœur, Paul (2004), Sur la traduction, Paris: Bayard. 
Regattin, Fabio (2004), 'Théâtre et traduction: un aperçu du débat théorique', L'Annuaire théâtral: revue québécoise d'études théâtrales, 36, pp. 156-71, http://www.erudit.org/revue/annuaire/2004/v/n36/041584ar.pdf. Consulté le 15 juin 2011.

Ronfard, Jean-Pierre (1985), 'Qu'est-ce que le théâtre?', Études littéraires, 18 :2, pp. 227-31, http://id.erudit.org/iderudit/500729ar. Consulté le 16 novembre 2011.

Sarajeva, Susam (2011), 'The translation and reception of multilingual films', http://ns1076.dizinc.com/index.php?option=com_k2\&view=item\&id=316:the-translationand-reception-of-multilingual-films-/-la-traduction-et-r\%C3\%A9ception-de-filmsmultilingues\&Itemid=66. Consulté le 20 avril 2012.

Stratford, Madeleine (2008), 'Autour de Babel! Les défis multiples du multilinguisme', Meta: Journal des traducteurs, 53, pp. 457-70, http://www.erudit.org/revue/meta/2008/v53/n3/019234ar.pdf. Consulté le 2 février 2012.

Von Flotow, Luise (2000), 'Life is a caravanserai: translating translated Marginality, a Turkish-German Zwittertext in English', Meta: Journal des traducteurs, 45, pp. 65-72, http://www.erudit.org/revue/meta/2000/v45/n1/004578ar.pdf. Consulté le 11 juin 2012. Zatlin, Phyllis (2005), Theatrical Translation and Film Adaptation: A Practitioner's View, Clevedon: Multilingual Matters.

Zhang, X. (2006), 'Traduire le théâtre-Application de la théorie interprétative à la traduction des œuvres dramatiques françaises en chinois', Thèse de doctorat, Paris : Université Paris III- 
Sorbonne nouvelle ESIT, http://tel.archives-ouvertes.fr/.../these_zhang.pdf. Consulté le 10 novembre 2012.

\section{Contributor details}

Marcelline Massoua II is Lecturer in French at the University of Pretoria (South Africa). Originally from Cameroon, where geographical diversity echoes linguistic diversity as reflected in the literary production, she studied how translation could be used a means to reconcile linguistically separated individuals sharing the same territory and to breakdown the boundaries between languages and their users that, traditionally, seems to be fundamental to the very existence of translation. She is currently completing her Ph.D. research in the production and circulation of Cameroonian translated literature.

Contact:

University of Pretoria,

100 Duxbury Road, B24 Protea, Hatfield, Pretoria, 0083, South Africa.

E-mail: marcelline.massoua@up.ac.za

Notes

\footnotetext{
${ }^{1}$ Pour ne citer que quelques-unes: Johnston's (1996) Stages of Translation; Upton's (2000)

Moving Target; Baines, Marinetti and Perteghella's (2011) Staging and Performing

Translation.

${ }^{2}$ Sylvie Chalaye, professeur à l'Institut d'études théâtrales de Paris III et spécialiste de dramaturgie afro-caribéennes répondait aux questions de Jeune Afrique
} 
http://www.jeuneafrique.com/Article/ARTJAJA2534p090-092.xml1/sylvie-chalaye.-

Actualite_Info.html.

${ }^{3}$ Alain Ricard souligne une réflexion de Gunner sur l'hétéroglossie littéraire en Afrique du Sud et son caractère rhétorique: «heteroglossia [...] marks ' the act of wholesale translation into English' and gives a sense of the 'rhetoricity of the original', the 'rhetorical silence of the original'» (Gunner cité par Ricard 2004: 119).

${ }^{4}$ Ces références ne se limitent pas aux personnages. Et s'étendent par exemple à la résidence du président Akhikikriki dans Requiem for the Last Kaiser, si on inverse l'ordre des lettres de 'Iduote' on obtient Etoudi, où se trouve en réalité le palais présidentiel du Cameroun, le palais de l'unité. Il s'agit certainement d'une inversion symbolique.

${ }^{5}$ Pour un certain public francophone en dehors de l'Afrique.

${ }^{6}$ La version intégrale de la fameuse phrase est: «Vous souffrez, j'en suis conscient... J'ai confiance en vous. Faites-moi confiance...». Tels étaient les mots de consolation du Président Biya, le 31 décembre 1986, alors qu'il annonçait à la population camerounaise, déjà atteinte dans sa chair par une crise de trésorerie du pays imputable à la gabegie de la fonction publique, que le gouvernement n'avait plus d'autre choix que celui d'accepter le P.A.S du FMI, pour redresser la situation. Il faisait confiance aux Camerounais affamés de se serrer la ceinture afin de ne plus sentir la famine. En trois années seulement de gestion du Renouveau, et comme pour bien indiquer que sous ce règne les mots, notamment ceux de « rigueur » et de «moralisation » devaient désormais signifier pour tous exactement leur contraire, toutes les réserves monétaires du Cameroun à Paris, Genève et New York avaient fondu comme beurre au soleil (http://www.cameroonvoice.com/news/article-news-5276.html).

${ }^{7}$ De cette situation réelle au Cameroun est sortie une phrase célèbre du président, lorsqu'il est finalement arrivé à Douala quelques années plus tard; il a déclaré dans un discours: «Me 
voici donc à Douala » en réponse au défi lancé par l'opposition. Il était entouré de milliers de gardes armés jusqu'aux dents.

${ }^{8}$ Ce choix a été rejeté par les participants francophones à une conférence sur le multilinguisme qui s'est tenue à l'université de Pretoria en 2013 (GANAA 2013). Ceux-ci ont immédiatement reconnu la célèbre phrase du président de Gaulle et ont reproché à la traductrice ce changement.

${ }^{9}$ La Confrérie des Conards de Rouen: Passion généalogique (site web: http//passiongenealogique.hautefort.com/archives/2012/02/19/la-confrerie-des-conards-derouen.html). 